\title{
Trusted and Efficient Request Processing Using Packet List on Cloud
}

\author{
B.Deepika
}

\begin{abstract}
Distributed computing information is put away over the dispersed servers, which can be gotten to with the assistance of client questions. In distributed computing, a large number of client inquiries are handled by circulated server with less dormancy, high accessibility and high dependability. Be that as it may, giving privacy client inquiries is as yet a testing undertaking because of the high defenselessness exist in the cloud condition. The current components experience the ill effects of high computational and correspondence overhead, notwithstanding vulnerabilities still exists in their security instruments. In our proposed methodology, any client sends the spatial inquiries to the specialist co-op, at that point the specialist co-op utilize these spatial questions to keep an eye on the spatial scrambled information. Client utilizes the RC5 key to decode the reaction messages. In this whole procedure, clients can shield the information from the information spillage, diminishes the correspondence overhead when contrasted and other existing systems.
\end{abstract}

Keywords-Encryption key, service provider, user query, and decryption.

\section{INTRODUCTION}

Information proprietor of the unique information needs enormous number of computational and capacity assets when information proprietor gives the extraordinary information as an administration, this is certifiably not a down to earth approach for each datum proprietor. Answer for this issue is to send uncommon information in the cloud condition, which is practical methodology for each datum owner [1-5]. Spatial information redistributing is a developing region over the distributed computing. Indeed, even distributed computing is financially savvy approach, some security challenges likewise should be routed to shield the spatial information from the aggressors. The primary difficulties are anticipating information spillage; need trust on cloud specialist organization, and blocking unapproved access [6-10]. To deal with the above difficulties, information proprietor need to give classification, trustworthiness and accessibility in redistributed data.

Spatial information alludes to a wide range of information articles or components that are available in a topographical space or skyline. It empowers the worldwide finding and situating of people or gadgets anyplace in the world [10-15]. Spatial information is otherwise called geospatial information, spatial data or geographic data In our proposed methodology secure question handling is done among three modules, for example, Trusted User (TU), Service Provider (CSP) and Data Owner (DO). Our principle objective is to anchor the correspondence and keep up least the computational and correspondence overhead.

Revised Manuscript Received on 14 August, 2019.

B.Deepika ,Computer Science and Engineering, Malla Reddy Engineering College for Women, Hyderabad, India.1203.deepika@gmail.com

\section{PROPOSED SYSTEM}

To give high security to spatial inquiry preparing in distributed computing with least calculation and correspondence overhead, the current instruments are deficient, to beat this issue we have built up a spatial question handling by Hilbert spatial bend with lightweight encryption calculation. In our proposed methodology secure question handling is done among three modules, for example, Trusted User (TU), Cloud Service Provider (CSP) and Data Owner (DO).

Information Owner gives question preparing access rights to every single validated client through the specialist organization. Information proprietor utilizes Hilbert spatial bend to speak to the spatial information, in this methodology information proprietor figures the cell list and its comparing information purposes of spatial information by utilizing Hilbert spatial bend. Here, both file and information focuses are scrambled before it send to the SP, these encoded qualities additionally imparted to TU. DO utilizes RC5 encryption calculation to scramble the record and information purposes of Hilbert bundle list, this procedure decreases the computational overhead at information proprietor side and secures the information spillage to CSP and unapproved clients.

CSP has a rundown of TUs which are verified by DO, with that CSP gives spatial inquiry administration to every single confirmed client. Notwithstanding that, CSP just stores the scrambled spatial information and it doesn't have consent to unscramble the information. At first, it gets the scrambled information from the DO before it gives the support of any TU.

$\mathrm{TU}$ at first gets the file data about spatial question, utilize this data to produce ask for inquiry and send this inquiry to CSP. After getting this inquiry, CSP checks the client ask for is confirmed or not by checking the current accessible TU list. In the event that the client is a substantial, the client inquiry is prepared on scrambled spatial information. In light of the gained outcomes, CSP sends encoded spatial information as an answer message to client. At the point when client gets encoded answer from CSP, client decodes the answer by utilizing mystery RC5 key which is known to DO and TU. 


\section{Trusted and Efficient Request Processing using Packet List on Cloud}

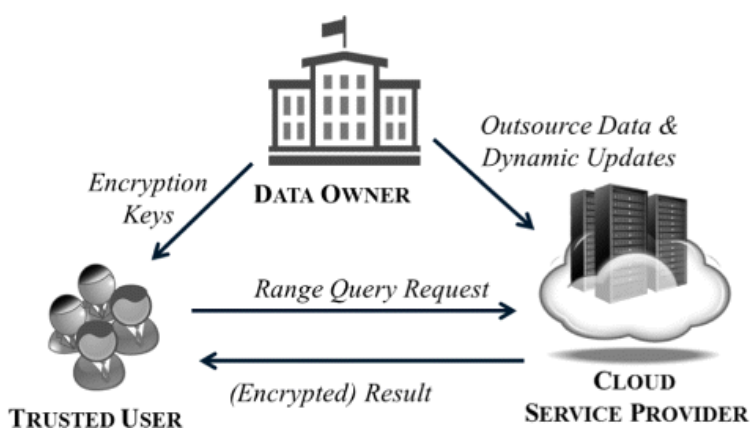

III. SySTEM ARCHITECTURE

The cloud engineering model utilized contains 3 fundamental substances, to be specific the Data Owner (DO), Cloud Service Provider (CSP) and Trusted User (TU). The DO ensures security by changing and encoding the spatial database before redistributing to the CSP. To change the 2D spatial information focuses, the DO utilizes the Hilbert space-filling bend. The DO shapes a rundown of bundles characterized by the Hilbert requesting. Next, this rundown is scrambled utilizing the OPE system, which permits spatial range inquiries to be performed at the CSP without drawing in the client and lessening any extra correspondence overhead. Moreover, the DO gives the Hilbert change key and in addition the encryption key to the TUs.

The keys are utilized by the TU to issue encoded extend questions to the CSP. The inquiry is handled on the encoded database at the CSP and the outcomes are come back to the TU. In conclusion, the TU decodes the inquiry reaction utilizing the encryption key to get the real outcome. In spatial database re-appropriating applications, the assailants must be kept from increasing unlawful access to the information. To dissect the security given by the proposed plans, it is expected that the clients are trusted by the information proprietors and, the change and encryption key is just given to the verified clients.

\section{Secure Range Query Processing Using Hilbert Packet List Algorithm}

Range query Input:

User's Trusted key, U $\mathrm{Ki}$

Range Data Points, $\mathrm{D}=\left(\mathrm{d}_{1}, \ldots \ldots, \mathrm{d}_{\mathrm{s}}\right)$

Packet Size, $\mathrm{P}_{\mathrm{s}}$

RC5 Encryption Key-K

Hilbert Packet List $=\left(\mathrm{P}_{1}, \ldots \ldots \ldots, \mathrm{P}_{\mathrm{p}}\right)$, where $\mathrm{P}_{\mathrm{i}}=\left[\mathrm{P}_{\mathrm{s}} ; \mathrm{P}_{\mathrm{e}} ; \mathrm{P}_{\mathrm{c}}\right]$

1)Data Owner:

Foreach $\mathrm{d}_{\mathrm{i}} \in \mathrm{D}$

Find minimum required space for each $d_{i}$

Compute cell index of $d_{i}$ using filled hilbert and add to $C$

2) Sort the list $\mathrm{C}$

3) foreach $c_{i} \in C$

$$
\mathrm{P}_{\mathrm{s}}=\mathrm{c}_{\mathrm{x}}
$$

$$
\begin{gathered}
\text { while size }\left(\mathrm{P}_{\mathrm{c}}\right)<\mathrm{k} \text { do } \\
\text { Add } \mathrm{d}_{\mathrm{j}} \text { to } \mathrm{P}_{\mathrm{c}}
\end{gathered}
$$

$\mathrm{P}_{\mathrm{e}}=\mathrm{c}_{\mathrm{y}}$

4) Data Owner: DO Encrypts Hilbert packet list with RC5 key and send to CSP

5) Data Owner: DO Sends Range query range and RC5 key to TU by encrypting the TU public key

6) Trusted User: TU requests the CSP by using range query

7) Cloud Service Provider: CSP sends encrypted range data to the TU as a reply
8) Trusted User: AU decrypts the range data by using known RC5 key

9) Cloud Service Provider: CSP sends alert message to DO, when it receives number false special query range requested from an TU.

\section{PERFORMANCE ANALYSIS}

In our proposed methodology, unapproved client can't get access from specialist co-op because of it doesn't have substantial key. Indeed, even the aggressor can break the validation by utilizing animal power approach still the assailant can't discover the mapping of encoded questions. On the off chance that number of false inquiries touched base at CSP, CSP is educated to DO comparing TU. DO will either erases TU from the CSP list or sends caution message to the TU. Then again, any Service supplier sends the phony information, TU identify the phony information effectively by utilizing RC5 unscrambling calculation.

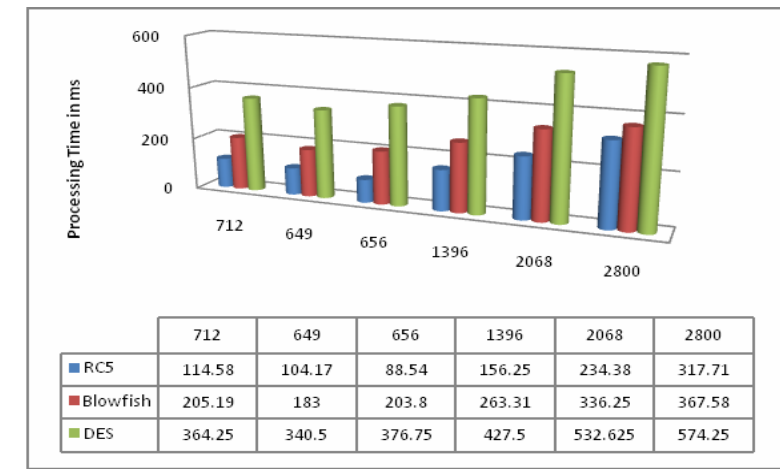

RC5 performs faster than Blowfish \& DES. shows the average execution time for these three algorithms to execute the files.RC5 is 1.54 times faster than Blowfish and 2.57 times faster than DES. Result also concludes that performance of Blowfish algorithm is inversely proportional to keysize, if keysize will increase the performance will decrease andvice-versa

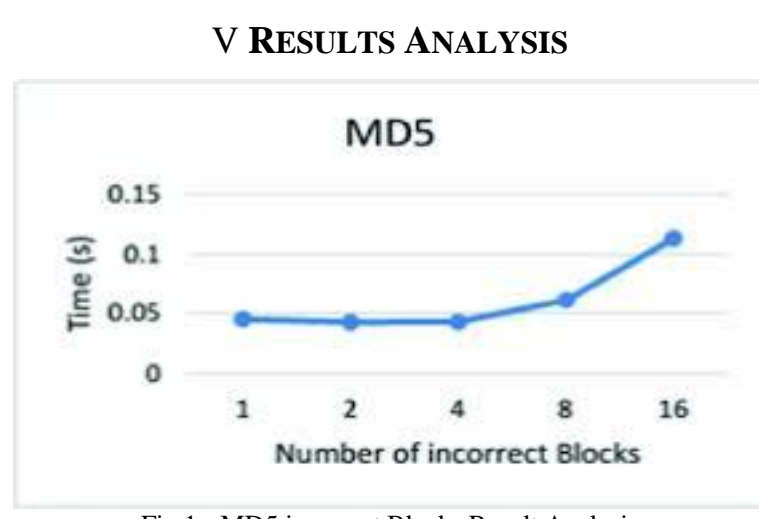

Fig 1:- MD5 incorrect Blocks Result Analysis 


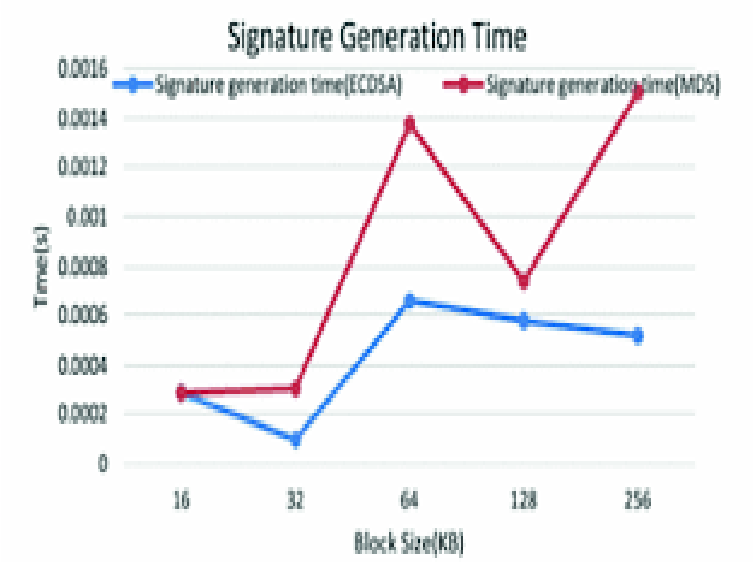

Fig 2:- Signature Generation Time

\section{CONCLUSION}

In this paper, we have proposed a protected spatial inquiry handling utilizing hilbert parcel list calculation to give secrecy, uprightness and accessibility to the spatial information, which is conveyed in the cloud condition. In light of our server, we found that the current systems experience the ill effects of high computational and correspondence overhead, notwithstanding vulnerabilities still exists in their security instruments. To diminish the computational overhead, we use RC5 keys to encode the spatial information, and to decrease the correspondence overhead, Data proprietor stores the scrambled spatial information in specialist organization. We have done the execution examination of proposed calculation in the antagonistic condition. In the long run, we found that our proposed calculation can shield against from information spillage, unapproved access and phony information recognizable proof assaults.

\section{REFERENCE}

1. Z. Xiao and Y. Xiao, "Security and protection in distributed computing," IEEE Communications Surveys and Tutorials,, vol. 15, no. 2, pp. 843$859,2013$.

2. M. L. Yiu, G. Ghinita, C. S. Jensen, and P. Kalnis, "Empowering look benefits on redistributed private spatial information," The VLDB Journal, vol. 19, no. 3, pp. 363-384, 2010.

3. P.Wang and C. V. Ravishankar, "Secure and effective range questions on re-appropriated databases utilizing r-trees," in 2013 IEEE 29th International Conference on Data Engineering (ICDE).IEEE, 2013, pp. 314- 325 .

A. M. Talha, I. Kamel, and Z. A. Aghbari, "Upgrading classification and protection of re-appropriated spatial information," in 2015 IEEE second International Conference on Cyber Security and Cloud Computing (CSCloud).IEEE, 2015, pp. 13-18.

4. S. Yu, C. Wang, K. Ren, and W. Lou, "Accomplishing secure, versatile, and fine-graineinformation get to control in distributed computing," in IEEE Infocom, 2010 procedures.IEEE, 2010, pp. 1-9.

5. H. Xu, S. Guo, and K. Chen, "Building private and proficient question benefits in the cloud with scratch information bother," IEEE exchanges on learning and information designing, vol. 26, no. 2, pp. 322-335, 2014.

6. H. Hu, J. Xu, C. Ren, and B. Choi, "Handling private inquiries over untrusted information cloud through security homomorphism," in IEEE 27th International Conference on Data Engineering.EEE, 2011, pp. $601-612$.

7. G. Zhao, C. Rong, J. Li, F. Zhang, and Y. Tang, "Believed information sharing over untrusted distributed storage suppliers," in IEEE Second International Conference on Cloud Computing Technology and Science (CloudCom). IEEE,2010,pp. 97- 103.
8. H. Hacigum" us, B. Iyer, and S. Mehrotra, "Giving database as a benefit," in eighteenth International Conference on Data Engineering, 2002. Procedures. IEEE,2002, pp. 29- 38

9. E. Damiani, S. Vimercati, S. Jajodia, S. Paraboschi, and P. Samarati, "Adjusting classification and productivity in untrusted social dbmss," in Proceedings of the tenth ACM gathering on Computer and Communications Security.ACM, 2003, pp. 93-102.

10. W.- S. Ku, L. Hu, C. Shahabi, and H. Wang, "Question honesty confirmation of area based administrations getting to redistributed spatial databases," in Advances in Spatial and Temporal Databases. Springer,2009, pp. 80- 97.

A. Khoshgozaran and C. Shahabi, "Private mate look: Enabling private spatial inquiries in informal organizations," in International Conference on Computational Science and Engineering, 2009 (CSE'09)., vol. 4. IEEE,2009, pp. 166- 173.

11. Anil Kumar Uppula,SrinivasuluTadisetty ," Achieving better Authentication and Copyright assurance Using DWT and SVD Based Watermarking Scheme, "International Journal of Computer Engineering In Research Trends.,vol.3,no.9,pp.487-491,September 2016.

12. VenkataSrinivasuVeesam, Bandaru Satish Babu," Evaluation of Captcha Technologies towards Graphical Password Scheme," International Journal of Computer Engineering In Research Trends.vol.2,no1,pp,98-106,February 2015.

13. D.J. AshpinPabi, N.Puviarasan, P.Aruna," Fast Singular esteem decay based picture pressure utilizing butterfly molecule swarm enhancement strategy (SVD-BPSO)," International Journal of Computer Engineering In Research Trends.,vol.4,no.4,pp.128-135,April 2017 